\title{
Three-dimensional analysis of heat flow, segregation, and interface shape of gradient-freeze crystal growth in a centrifuge
}

\author{
C.W. Lan*, C.Y. Tu \\ Department of Chemical Engineering, National Taiwan University, Taipei, Taiwan 10617, ROC \\ Received 16 February 2001; accepted 2 April 2001 \\ Communicated by D.T.J. Hurle
}

\begin{abstract}
Three-dimensional (3D) heat flow, dopant segregation, and interface shape during crystal growth by a gradient freeze technique in a centrifuge are analyzed by a finite volume method. The basic flow patterns for a fixed geometry (with a concave interface) at different configurations agree well with the previous report (Friedrich et al., J. Crystal Growth 167 (1996) 45). However, the self-consistent analysis allows us for the first time to further investigate the role of the Coriolis force and centrifugal acceleration on the heat and mass transfer and the interface, simultaneously. Furthermore, the rotation speed found for the weakest convection, where the Coriolis force balances the gravitational and centrifugal forces, turns out to have larger radial segregation, despite having a larger effective segregation coefficient. Rotation about the growth axis is also investigated. For this configuration, it is found that both axial and radial segregation could be reduced under certain conditions. (C) 2001 Elsevier Science B.V. All rights reserved.
\end{abstract}

PACS: 44.25. +f; 47.27.Te; 81.10.Fq; 02.60.c6; 02.70.Fj

Keywords: A1. 3D computer simulation; A1. Segregation; A1. Interface shape; A2. Coriolis force; A2. Centrifugal acceleration; A2. Gradient freeze

\section{Introduction}

The control of melt flow, which influences the interface morphology and dopant segregation (both radial and axial), is important in bulk crystal growth. The most effective way for the flow and thus segregation control is by external forces. Crystal growth in a centrifuge has been regarded as one way for growth control (e.g. [1,2]). A minimum segregation (with the largest effective segregation coefficient) at the so-called "magic-g

\footnotetext{
*Corresponding author. Tel./fax: + 886-2-23633917.

E-mail address: lan@ruby.che.ntu.edu.tw (C.W. Lan).
}

level" was experimentally found by Regel and Rodot [2]. Furthermore, Müller et al. [3] have shown, both experimentally and numerically, that the Coriolis force suppresses the oscillatory convection in top-seeded Bridgman growth. Similar observations were also found by Rodot et al. [2]. Since then, much attention has been paid to find the magic-g level for the minimum convection, which may be a condition for minimum segregation (e.g. [3-6]), i.e., the diffusion-controlled limit being approached. Thus, this condition would resemble microgravity as well. However, the numerical simulation in these studies was focused on the heat transfer in the melt only. Segregation 
and interface morphology were not considered. The axial segregation was estimated by a simplified model based on the boundary-layer theory. More importantly, because of the poorer mixing at the magic-g level, the radial dopant distribution of a grown crystal in a centrifuge could be an important issue, but it has not yet been discussed. Besides the use of a centrifuge, steady ampoule rotation has a similar effect. In a recent study by Lan [7], it was found that an inversion of radial segregation exists at a certain rotation rate for the growth with a concave interface. At this critical rotation rate, a flow transition was also illustrated numerically, where the centrifugal acceleration becomes dominant. In addition, a more diffusion-controlled axial segregation could be achieved, where the effective segregation coefficient was closer to one. However, there is a disadvantage due to the steady ampoule rotation under asymmetric heating. The analysis by Lan et al. [8] has shown that severe angular segregation can be induced. Therefore, on the other hand, it is believed that the use of a centrifuge, carrying the whole growth system, may be more feasible in practice. In fact, with axisymmetric heating, the steady ampoule rotation is equivalent to the situation in a centrifuge by placing vertically the sample at the rotating axis.
In this study, we present for the first time a selfconsistent simulation of a gradient-freeze growth in a centrifuge. Before the full model is taken into account, we illustrate first the basic flow structures at different configurations and rotation speeds with a fixed interface and further compare our results with the excellent work done by Müller's group [1]. Starting from there, the interface is then solved with the field variables simultaneously, so that the convection induced by interface deformation can be better simulated. Interface concavity and growth rate are also discussed. Furthermore, $3 \mathrm{D}$ radial segregation patterns are calculated and the condition for reducing segregation is discussed.

\section{Model description and numerical solution}

A generic gradient-freeze crystal growth system in a centrifuge is illustrated in Fig. 1a. Since the configuration is truly three-dimensional (3D), the system is described by Cartesian coordinates $(x, y, z)$ in a rotating frame with an angular rotation vector $\vec{\Omega}$. For comparison purposes, the ambient thermal distribution is set to be linear with a constant gradient $G$. Although the fully time-dependent calculation is carried out here, the simulation of a whole growth period is still too

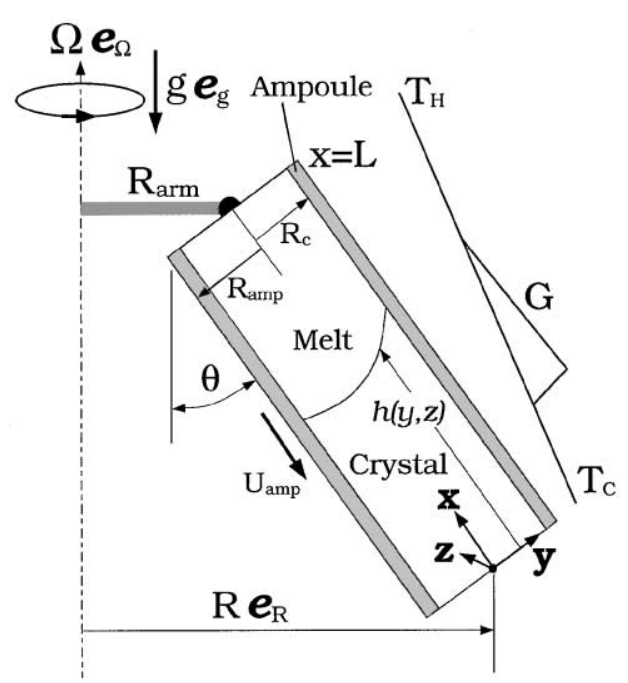

(a)

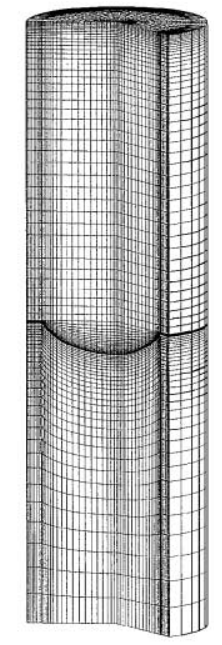

(b)

Fig. 1. (a) Schematic sketch of gradient-freeze growth in a centrifuge; (b) a portion of a sample mesh used in the calculations. 
costly. Therefore, for the calculation of dopant fields, we have further adopted a pseudo-steadystate approximation, with the ampoule pulling speed at $U_{\text {amp. }}$ The averaged growth rate is set at the same speed. It should be pointed out that in practice the furnace temperature decreases with time for gradient freeze, i.e., $U_{\mathrm{amp}} G$ being the cooling rate. Therefore, the present treatment is just a simple way to treat the pseudo-steady situation. The time-dependent calculation is then imposed to the steady state to reveal the transient behavior. Similar treatment has been adopted by many authors for two-dimensional analyses (e.g., $[9,10])$, and the calculated radial dopant distribution is consistent with that by a global timedependent analysis [11].

The flow, temperature, and dopant fields as well as the melt/crystal interface are also represented in the Cartesian coordinate $(x, y, z)$. As shown in Fig. 1a, the centrifuge has an arm $R_{\text {arm }}$ and the real radius to any point in the system can be determined if the tilt angle $\theta$ is set. For the freeswing configuration, if the center of mass of the system is at the origin of the coordinate system, the steady angle $\theta$ of the furnace is given by $R \Omega^{2}=$ $g \tan (\theta)$ with $R=R_{\text {arm }}+L \sin (\theta)$, where $g$ is the gravitation acceleration and $L$ the length of the sample. For this case, the resultant gravity is thus antiparallel to the $x$-axis. Furthermore, it is also assumed that the dopant concentration is so low that its effects on the flow and liquidus temperature can be neglected.

Since the origin is defined at the bottom of the ampoule, the rotation arm vector $\vec{R}$, angular rotation vector $\vec{\Omega}$, and gravity vector $\vec{g}$ are defined as follows: $\vec{R}=R \vec{e}_{R}=R\left[-\sin (\theta) \vec{e}_{x}+\cos (\theta) \vec{e}_{y}\right]$, $\vec{\Omega}=\Omega \vec{e}_{\Omega}=\Omega\left[\cos (\theta) \vec{e}_{x}+\sin (\theta) \vec{e}_{y}\right], \vec{g}=g \vec{e}_{g}=g[-$ $\left.\cos (\theta) \vec{e}_{x}-\sin (\theta) \vec{e}_{y}\right]$, where $\vec{e}_{x}, \vec{e}_{y}$, and $\vec{e}_{z}$ are the unit vectors in the $x$-, $y$-, and $z$-direction, respectively. At any point on the sample, the position vector is given by $\vec{r}=x \vec{e}_{x}+y \vec{e}_{y}+z \vec{e}_{z}$. Then, the resultant acceleration vector $\vec{b}$ can be represented as:

$\vec{b}=\vec{g}-[\vec{\Omega} \times(\vec{\Omega} \times(\vec{r}+\vec{R}))]$.

Furthermore, the dimensionless variables are defined by scaling length with the crystal diameter
$D_{\mathrm{c}}$, velocity with $\alpha_{\mathrm{m}} / D_{\mathrm{c}}$, pressure with $\rho_{\mathrm{m}} \alpha_{\mathrm{m}}^{2} / D_{\mathrm{c}}^{2}$, and dopant concentration with its average concentration $C_{0}$ in the crystal, where $\alpha_{\mathrm{m}}$ is the thermal diffusivity and $\rho_{\mathrm{m}}$ the melt density. The dimensionless temperature is scaled by the melting point $T_{\mathrm{m}}$. Based on the rotating frame with the angular velocity $\vec{\Omega}$, the time-dependent governing equations describing convection and heat and dopant transport in the melt (m) are as follows:

$$
\nabla \cdot \vec{v}=0,
$$

$$
\begin{aligned}
\frac{\partial \vec{v}}{\partial t}+\vec{v} \cdot \nabla \vec{v}= & -\nabla p+\operatorname{Pr} \nabla \vec{v}-\operatorname{PrRa}_{\mathrm{T}}(T-1) \vec{e}_{b} \\
& -\operatorname{PrTa}^{1 / 2} \vec{e}_{\Omega} \times \vec{v},
\end{aligned}
$$

$\frac{\partial T}{\partial t}+\vec{v} \cdot \nabla T=\nabla^{2} T$,

$\frac{\partial C}{\partial t}+\vec{v} \cdot \nabla C=\frac{\operatorname{Pr}}{\mathrm{Sc}} \nabla^{2} C$,

where $\vec{v}, t, \not, P$ and $C$ are the dimensionless velocity, time, modified pressure, temperature, and dopant concentration, respectively. $\mathrm{Pr}$ is the Prandtl number $\left(\operatorname{Pr} \equiv v_{\mathrm{m}} / \alpha_{\mathrm{m}}\right)$ and Sc the Schmidt number $\left(\mathrm{Sc} \equiv v_{\mathrm{m}} / D\right)$, where $v_{\mathrm{m}}$ is the kinematic viscosity and $D$ the dopant diffusivity in the melt. In Eq. (3), the thermal Rayleigh number $\mathrm{Ra}_{T}$ and the Taylor number $\mathrm{Ta}$ in the source term are defined as follows:

$\mathrm{Ra}_{\mathrm{T}} \equiv \frac{g \beta_{\mathrm{T}} T_{\mathrm{m}} D_{\mathrm{c}}^{3}}{\alpha_{\mathrm{m}} v_{\mathrm{m}}^{2}} ; \quad \mathrm{Ta}=4 \Omega^{2} D_{\mathrm{c}}^{4} / v_{\mathrm{m}}^{2}$,

where $\beta_{\mathrm{T}}$ is the thermal expansion coefficient. If one is interested in using temperature difference to represent the Rayleigh number, the conversion is also straightforward. Furthermore, the dimensionless resultant acceleration $\vec{e}_{b}$ becomes

$\vec{e}_{b}=\vec{e}_{g}-\operatorname{Fr}\left[\vec{e}_{\Omega} \times\left(\vec{e}_{\Omega} \times(\vec{r}+\vec{R})\right]\right.$,

where the Froude number $\mathrm{Fr}=\Omega^{2} D_{\mathrm{c}} / g$ is the ratio of centrifugal and gravitational accelerations; $\vec{r}$ and $\vec{R}$ are dimensionless here. Furthermore, in Eq. (3) $\operatorname{PrTa}^{1 / 2} \vec{e}_{\Omega} \times \vec{v}$ is the dimensionless Coriolis force. The static pressures due to gravitational and centrifugal accelerations are combined with the original pressure when using the Boussinesq approximation. 
In the crystal (c) and the ampoule (a), only heat transfer needs to be considered:

$\frac{\partial T}{\partial t}+\mathrm{Pe}_{i} \vec{e}_{x} \cdot \nabla T=\kappa_{i} \nabla^{2} \theta,(i=\mathrm{c}, \mathrm{a})$,

where $\mathrm{Pe}_{i} \equiv \rho_{i} C p_{i} U_{\mathrm{amp}} D_{\mathrm{c}} / k_{\mathrm{m}}$ is the Peclect number, $\kappa_{i} \equiv k_{i} / k_{\mathrm{m}}$ the dimensionless thermal conductivity of crystal or ampoule: $k_{\mathrm{m}}$ is the thermal conductivity of the melt, $\rho_{i}, C p_{i}$, and $k_{i}$ are the density, specific heat, and thermal conductivity of the phase $i(i=c$ or $a$ ), respectively.

Suitable boundary conditions are also necessary. The no-slip condition is used for the melt velocity on solid boundaries. When the pseudosteady-state is assumed, the upper open boundary is considered as an artificial boundary $[9,10]$, and its velocity boundary condition is kept the same. The thermal and solutal boundary conditions at the melt/solid interfaces are set by the heat and mass flux balances. For example, at the growth front:

$$
\begin{aligned}
& \left.\vec{n} \cdot \nabla T\right|_{m}-\left.\vec{n} \cdot \kappa_{\mathrm{c}} \nabla T\right|_{\mathrm{c}} \\
& \quad+\gamma_{c}\left(\mathrm{Pe}_{\mathrm{m}}-\frac{\partial h}{\partial t}\right) \operatorname{St}\left(\vec{e}_{x} \cdot \vec{n}\right)=0, \\
& \left.\vec{n} \cdot \nabla C\right|_{m} \\
& \quad+\gamma_{c}\left(\mathrm{Pe}_{\mathrm{m}}-\frac{\partial h}{\partial t}\right) \frac{\mathrm{Sc}}{\operatorname{Pr}}(1-K) C\left(\vec{e}_{x} \cdot \vec{n}\right)=0,
\end{aligned}
$$

where $\vec{n}$ is the unit normal vector at the growth interface pointing to the melt. The Stefan number $\mathrm{St} \equiv \Delta H /\left(C p_{\mathrm{m}} \Delta T\right)$ scales the heat of fusion $(\Delta H)$ released during solidification to the sensible heat in the melt; $\gamma_{\mathrm{c}} \equiv \rho_{\mathrm{c}} / \rho_{\mathrm{m}}$ is the ratio of density in the crystal over the melt. The equilibrium segregation coefficient $K$ of the solute is according to the phase diagram; $K \equiv C_{\mathrm{c}} / C$ at the growth interface, where $C_{\mathrm{c}}$ is the dopant concentration in the crystal. The temperature at the melt/solid interface is assumed to be the melting point. In Eq. (9), the dopant diffusion in the solid phases is ignored.

For simplicity, the temperatures at the top and bottom surfaces are set to be the furnace temperatures. With the pseudo-steady-state assumption, an artificial boundary condition is used at the upper boundary for the consistence of the overall dopant balance [8,9]. The heat exchange between the ampoule and the furnace is by both radiation and convection according to the energy balance along the ampoule surface,

$$
-\left.\vec{n} \cdot \kappa_{\mathrm{a}} \nabla T\right|_{\mathrm{a}}=\operatorname{Bi}\left(T-T_{\mathrm{a}}\right),
$$

where $\vec{n}$ is the unit normal vector on the ampoule surface pointing outwards, $\mathrm{Bi} \equiv h D_{\mathrm{c}} / k_{\mathrm{m}}$ the Biot number. For simplicity, the radiation effect is incorporated into the enhanced heat transfer coefficient $h$. The effective furnace temperature $T_{\mathrm{a}}$ is assumed to be linear with the gradient $G$; it is equal to $T_{\mathrm{m}}$ at $x=L / 2$.

The above governing equations and their associated boundary conditions can only be solved numerically. We have developed an efficient finite volume method (FVM) scheme using the primitive variable formulation [12] and multigrid acceleration [13] for the free boundary problem. This approach is much more efficient and robust than the previous FVM/Newton's method [14]. A sample converged mesh for calculation is shown in Fig. 1b. As shown, finer grid spacing is placed near the boundaries to enhance the accuracy of calculation. Two grid levels are used. In the first level, there are $10 \times 18 \times 30$ (in the radial, angular, and axial directions, respectively) finite volumes in the melt and $10 \times 18 \times 15$ in the crystal, and $5 \times 18 \times 45$ in the ampoule. The second level doubles the finite volumes in each direction, i.e., $20 \times 36 \times 60$ cells in the melt. All calculations are obtained by the fine grid. The calculations are performed in personal computers (AMD/K7$800 \mathrm{MHz}$ CPU with $512 \mathrm{M}$ SRAM). One calculation takes about $2 \mathrm{~h}$ of CPU time. It should be pointed out that the convergence of the steadystate approach is much slower and requires significant relaxation due to the large Coriolis force term. Therefore, the simulation is conducted through a fully time-dependent calculation using an implicit scheme. The integration step is less than $0.2 \mathrm{~s}$. Usually, a steady-state result can be easily obtained in 10-40 s, but all the calculated results are taken after $100 \mathrm{~s}$. Detailed description of the numerical method can be found elsewhere [13].

To evaluate the axial segregation, we use the effective segregation coefficient $K_{\text {eff }}$ defined by 
Adornato and Brown [9], i.e.,

$K_{\text {eff }} \equiv 1 /\langle C\rangle$,

where $\langle C\rangle$ is the average dopant concentration in the melt. Although this value differs from the one obtained by the best-fit from the axial segregation, it provides a good index for the global dopant mixing. If the mixing is poor (say for a diffusionlimited situation), the dopant concentration is near $C_{0}$ (or 1 for dimensionless concentration). Then, $K_{\text {eff }}$ is close to one. For such a case, if the growth length is long enough (much longer than the diffusion length), the axial segregation is diminished. On the contrary, if the global mixing is good, the dopant concentration is uniform being $C_{0} / K$. Then, $K_{\text {eff }}=K$, which is the equilibrium value obtained from the phase diagram.

\section{Results and discussion}

For comparison purposes, we consider mostly the gallium-doped germanium $(\mathrm{GaGe})$ growth in the gradient-freeze furnace investigated by Friedrich et al. [1] in his study. They considered the cases with a fixed interface and the calculations were restricted to the melt only. Two cases for gallium melt were also considered. However, to simulate the similar system in a self-consistent manner, we have included the free interface at the center of the domain, as shown in Fig. 1a. The position and shape of the interface are affected by the global heat transfer in the system as well as by the melt convection. The physical properties and some input parameters used are listed in Table 1. For comparison purposes, the radiation effect is not considered here for keeping the thermal profile inside the sample as linear as possible.

\subsection{Basic flow structures}

We have performed a series of calculations for the comparison with the previous results [1] considering different growth configurations and rotation speeds. Since the exact interface deflection used in the previous report is not available, we need to find a reference state to start the comparison. To do this, we first calculate the case
Table 1

Physical properties and some input parameters $[9,17]$

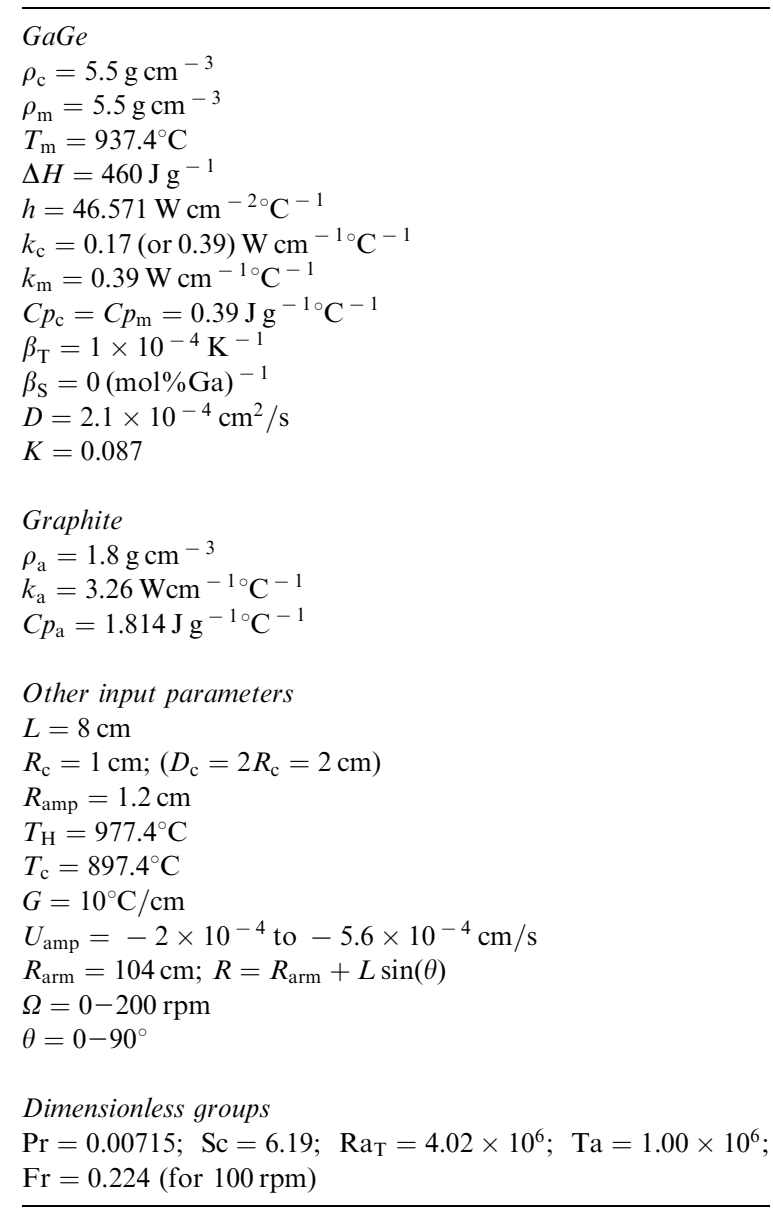

for no convection. By adjusting (increasing) the thermal conductivity of the crystal, we get a smaller deflection of interface, which matches the radial thermal gradient used by Friedrich et al. [1] for getting the same convection level at normal gravity $(0 \mathrm{rpm})$. Then, the interface is frozen for the rest of the calculations. The calculated samples for this configuration with a free-swinging angle are illustrated in Fig. 2. As shown for the cases of 0,40 , and $80 \mathrm{rpm}$, the basic flow structures in the $x-z$ plane are in good agreement with that presented by Friedrich et al. [1]. For 0 rpm shown in Fig. $2 \mathrm{a}\left(\theta=0^{\circ}\right)$, the flow is axisymmetric and its structure is typical for the gradient-freeze configuration with a concave interface $[9,10]$. At $40 \mathrm{rpm}$ 


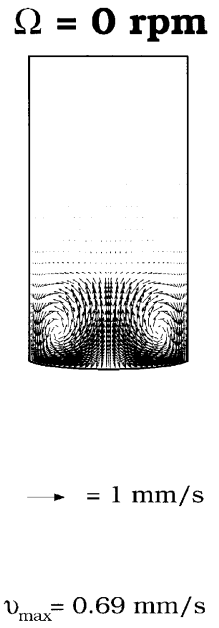

(a)

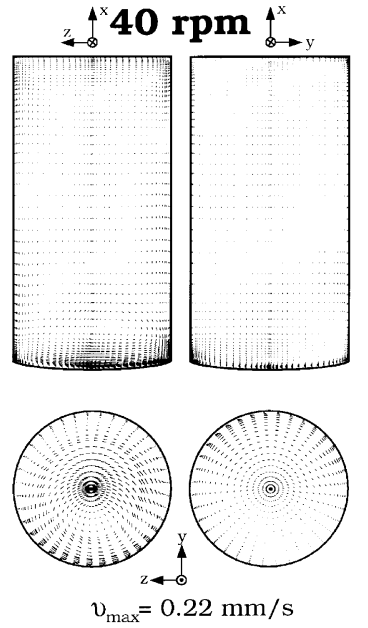

(b)

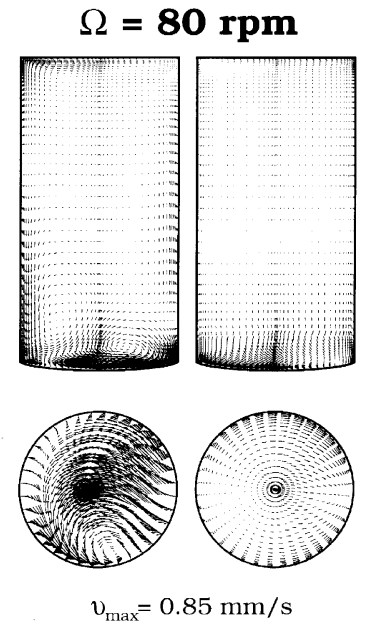

(c)

Fig. 2. Calculated results for small interface deflection (fixed) similar to the ones used by Friedrich et al. [1]: (a) $0 \mathrm{rpm}\left(\theta=0^{\circ}\right)$; (b) $40 \mathrm{rpm}\left(\theta=63.32^{\circ}\right)$; (c) $80 \mathrm{rpm}\left(\theta=82.89^{\circ}\right)$.

$\left(\theta=63.32^{\circ}\right)$, the flow near the growth interface is significantly suppressed by the Coriolis force and the flow structure is also changed dramatically. As it will be discussed shortly, this significantly alters the radial dopant distribution. Although the averaged resultant gravity direction is still antiparallel to the growth axis, the centrifugal acceleration and the Coriolis force in the melt are asymmetric leading to the 3D flow. Due to the nonuniform forces, the global convection increases slightly away from the interface. As the rotation speed is further increased to $80 \mathrm{rpm}\left(\theta=82.89^{\circ}\right)$, the centrifugal force becomes dominant and the convection increases, which can be seen from the larger velocity vectors. The flows in the $x-y$ plane are also shown, but they are in general featureless except for the flow near the growth interface.

We also present two flow patterns in the $y-z$ plane shown at the bottom of the figures for 40 and $80 \mathrm{rpm}$, respectively. One is at $x=4.2 \mathrm{~cm}$ and the other at $4.9 \mathrm{~cm}$. The edge of the interface is about at $x=4.0 \mathrm{~cm}$. As shown, near the interface the flow is mostly counterclockwise, but at some place the flow may be in the opposite direction. Interestingly, at $80 \mathrm{rpm}$, the flow pattern at $x=$ $4.9 \mathrm{~cm}$ shows two cells with different flow directions. Therefore, the flow seen from the top does not have a well-defined structure. Closer to the growth interface, the counterclockwise flow seems to be more clear. Nevertheless, as the interface becomes flat, the flow pattern is also changed. The orientations for the figures in Fig. 2 will be used for the rest of study, unless otherwise stated.

The maximum melt velocity varying with the rotation speed is further illustrated in Fig. 3 (open symbols), where the results of Friedrich et al. [1] (filled symbols and solid lines) are also included for comparison. We also perform calculations for gallium melt (both the free-swing and horizontal configurations). The comparison with the previous study is also shown in Fig. 3. As shown, they are all in good agreement. The solid lines in Fig. 3 are from the scaling analysis of Friedrich et al. [1]. As shown for the free-swing case of $\mathrm{Ge}$, there is a minimum of convection at about $20 \mathrm{rpm}$. This is supposed to be the so-called magic-g level having the least axial dopant segregation. At this critical rotation rate, the Coriolis force balances the two gravitational forces. Beyond this value, the centrifugal force becomes dominant and enhances the convection.

For the case with a flat bottom, there is no flow at $0 \mathrm{rpm}$ (at $\theta=0^{\circ}$ or vertical orientation) because the gravitational force is perfectly antiparallel to the thermal gradient. Therefore, as shown at the lower plot of Fig. 3, the maximum velocity 


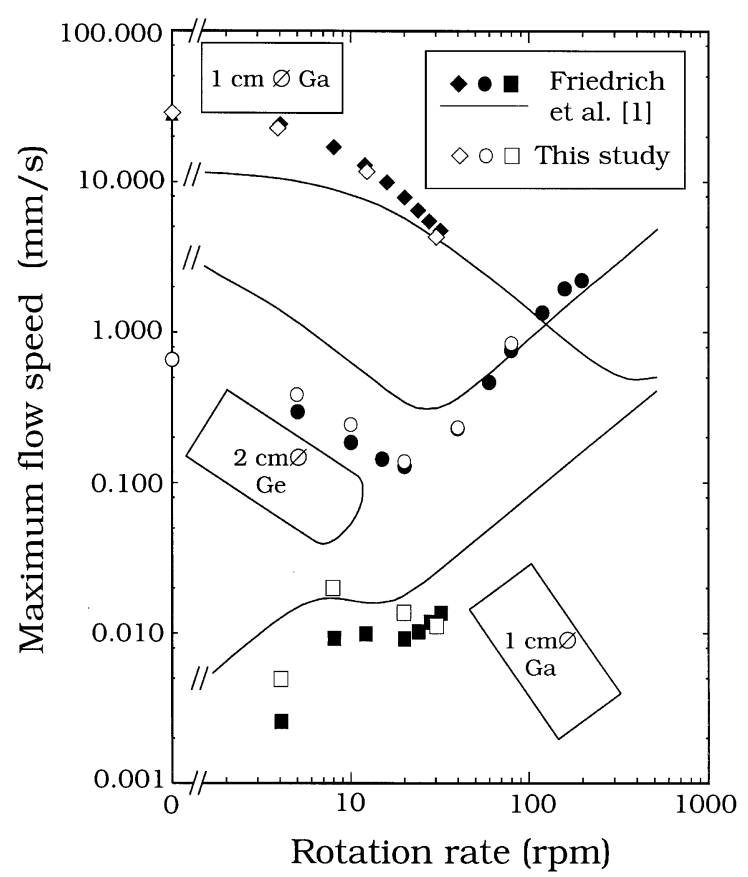

Fig. 3. Effects of rotation rate on the maximum melt velocity for various growth configurations. Present results are indicated by the open symbols and the filled symbols and the solid lines are results from Ref. [1].

increases with the rotation first and then decreases before the centrifugal force becomes dominant. On the other hand, the flow speed in the horizontal configuration, shown at the top of Fig. 3, decreases monotonically with the rotation speed. In this case without rotation, convection is quite strong because gravity is perpendicular to the axial thermal gradient. The maximum melt velocity is more than one order of magnitude higher than that in the vertical case. Nevertheless, in this configuration the flow in the Ga melt is still stable. However, for the 2-cm Ge growth oscillatory flows can be easily induced, which will be illustrated in Section 3.2.

\subsection{Interface deflection and segregation}

As we allow the interface to deform using the crystal thermal conductivity reported in the literatures (e.g. $[9,15])$, the interface becomes much more concave toward the melt. This is typical for a linear heating profile [15]. For the free-swing configuration, the distortion of the isotherms and also the bending of the interface are small due to the weaker flow and the small Prandtl number (0.00715). However, for the horizontal case the interface deformation is significant. Fig. 4 shows the effects of rotation on the melt flow and the interface shape for the horizontal configuration $\left(\theta=90^{\circ}\right)$. The ampoule pulling speed is set to $-2 \times 10^{-4} \mathrm{~cm} / \mathrm{s}$. Without rotation the flow is unsteady and only a snap shot is shown in Fig. 4a. The oscillation is quite random and the periods are smaller than $1 \mathrm{~s}$. As shown, significant buoyant flow is induced because gravity orientation is perpendicular to the thermal gradient. The flow in the $x-y$ plane is mainly in clockwise direction. The isotherms and also the interface shape are significantly distorted by the flow. Due to the unsteady nature, the flow near the centerline is not stable. Furthermore, the flow seen from the bottom (at the $x-z$ plane) does not remain symmetric about the centerline due to nonlinear symmetry breaking. The maximum melt speed is about $30 \mathrm{~mm} / \mathrm{s}$.

As the rotation speed is increased to $30 \mathrm{rpm}$, the flow becomes weaker and stable. Now the maximum melt velocity is only $1.79 \mathrm{~mm} / \mathrm{s}$. As shown in Fig. $4 b$, the distortion of the isotherms is greatly reduced. As a result, the interface becomes almost axisymmetric. Interestingly, the flow structures at both views at $30 \mathrm{rpm}$ turn out to be similar to the ones shown in Fig. 2b (the free-swing case). Again, beside the similar flow structures, the maximum flow speeds of both cases become closer as the rotation speed is further increased.

For the free-swing configuration, similar calculations using the real crystal thermal conductivity are carried out. The calculated results, which are similar to that of Fig. 2, are shown in Fig. 5, where the dopant fields (iso-concentration lines) are also included. Due to the small Pr number and the weak flow, the effect of flow on the isotherms and the interface shape for the free-swing case is small. Therefore, the larger interface concavity, compared with that in Fig. 2, is due to the lower crystal thermal conductivity used here [15]. Although the use of the same crystal and melt conductivity gives a much flatter interface, which is closer to the 


\section{$\Omega=\mathbf{0}$ rpm}
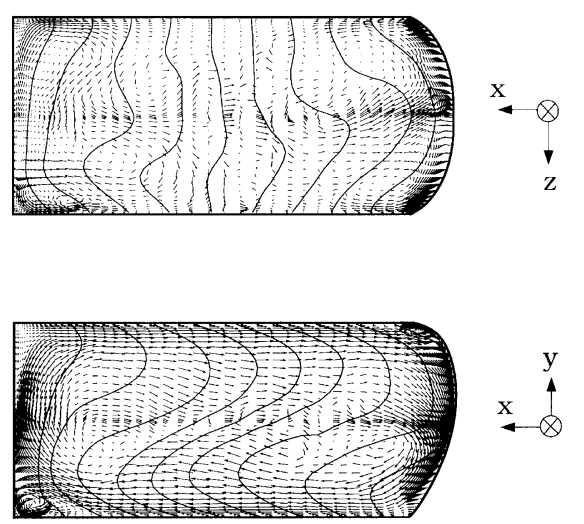

$$
\begin{aligned}
\longrightarrow & =6.67 \mathrm{~cm} / \mathrm{s} \\
v_{\max } & =30.21 \mathrm{~mm} / \mathrm{s}
\end{aligned}
$$

(a)

\section{$\Omega=30 \mathrm{rpm}$}
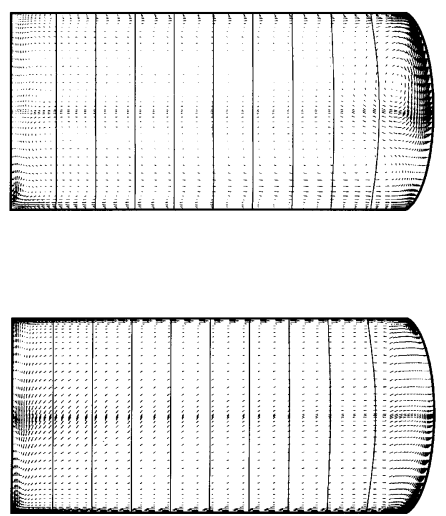

$$
\theta=90^{\circ}
$$

$\longrightarrow=4 \mathrm{~mm} / \mathrm{s}$

$v_{\max }=1.79 \mathrm{~mm} / \mathrm{s}$

(b)

Fig. 4. Effects of rotation on the interface, isotherms, and flow patterns for the horizontal configuration $\left(\theta=90^{\circ}\right)$ : (a) $0 \mathrm{rpm}$; $(\mathrm{b})$ $30 \mathrm{rpm} . \Delta T=\left(T_{\mathrm{H}}-T_{\mathrm{m}}\right) / 10$.

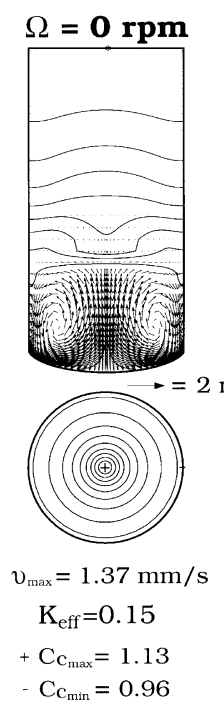

(a)

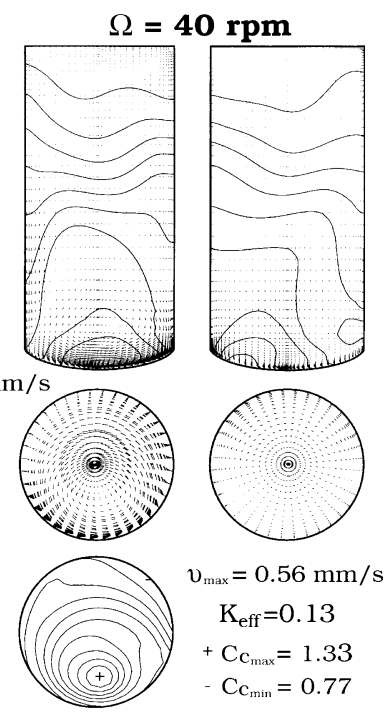

(b)

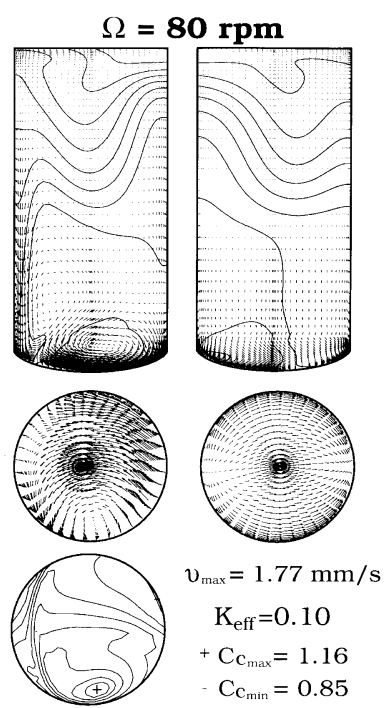

(c)

Fig. 5. Effects of rotation rate on the flow and dopant fields for the free-swing configuration: (a) $0 \mathrm{rpm}\left(\theta=0^{\circ}, C_{\mathrm{min}}=1.82\right.$, $\left.C_{\max }=12.94\right)$; (b) $40 \mathrm{rpm}\left(\theta=63.32^{\circ}, C_{\min }=2.58, C_{\max }=15.27\right)$; (c) $80 \mathrm{rpm}\left(\theta=82.89^{\circ}, C_{\min }=4.58, C_{\max }=13.32\right)$; bottom figures are the dopant distributions in the grown crystal. $U_{\mathrm{amp}}=-2 \times 10^{-4} \mathrm{~cm} / \mathrm{s}, \Delta C=\left(C_{\max }-C_{\min }\right) / 10$, and $K_{\mathrm{eff}}=1 /\langle C\rangle$.

experimental observation [1], the value used for benchmarking $[9,10]$ is adopted here. We believe that the thermal gradients in the furnace used in the experiment were higher in the crystal and lower in the melt. Interestingly, this argument has been confirmed in Friedrich's thesis [16]. Besides the stronger thermal convection for the more concave interface, the flow and dopant fields based on 
different concavities are similar, except that for the flat interface.

Due to the large Sc value, the dopant transport is mainly driven by the flow. Therefore, the dopant fields in Fig. 5 are significantly affected by the flow. For the case without rotation shown in Fig. 5a, the dopant distribution in the grown crystal is axisymmetric. In the figures, $C_{\min }$ and $C_{\max }$ are the minimum and maximum dopant concentrations in the melt, while $C_{\mathrm{c}_{\min }}$ and $C_{\mathrm{c}_{\max }}$ are the minimum and maximum dopant concentration in the grown crystal; $C_{\mathrm{c}}=K C$. Since the flow is induced locally near the growth front, the dopant fields are less distorted and the mixing is less away from the interface. Due to the slow growth rate $\left(2 \times 10^{-4} \mathrm{~cm} / \mathrm{s}\right)$, the effective segregation coefficient $K_{\text {eff }}$ is 0.147 which is not large. As the rotation rate is increased to $40 \mathrm{rpm}$, the flow near the interface is suppressed leading to poorer dopant mixing. Accordingly, the radial segregation in the grown crystal increases dramatically. Nevertheless, since the global dopant mixing is improved, the effective segregation coefficient decreases slightly. In this case, $K_{\text {eff }}$ is the largest at about $20 \mathrm{rpm}$. However, due to the low growth rate, we do not anticipate much improvement in the axial segregation here by the centrifuge. But, as the growth rate increases, the increase of $K_{\text {eff }}$ by rotation becomes significant, which will be dis- cussed shortly in Fig. 7. Further increasing the rotation speed to $80 \mathrm{rpm}$, both local and global mixings increase, because a stronger flow arises by the centrifugal acceleration. As a result, the radial segregation decreases. Meanwhile, $K_{\text {eff }}$ also decreases quite significantly. The peak of the dopant distribution in the grown crystal is located closer to the axis of rotation (opposite to the $y$-direction). Again, as just mentioned, the flow near the growth interface is counterclockwise. Therefore, the distortion of the dopant distribution in the crystal is also driven in the same direction.

Although the flow structures in Figs. 2 and 5 are similar, the interface shape plays an important role in the local flow. To illustrate this, we have performed additional calculations for the flat and slightly concave interfaces, shown in Fig. 6, for free-swing rotation at $40 \mathrm{rpm}$. In Fig. $6 \mathrm{a}$, the interface shape is fixed (flat). Interestingly, the flow direction near the growth front is reversed, both in the $x-z$ and $x-y$ planes. As a result, the peak dopant concentration appears at the edge, while the concentration is lower at the center. The radial segregation is also smaller. As the interface is allowed to move for $k_{\mathrm{c}}=k_{\mathrm{m}}$, the interface deflection is mainly due to the heat of fusion released by the growth. Then the flow pattern becomes similar to that shown in Fig. $2 b$. Although the maximum melt speed remains about

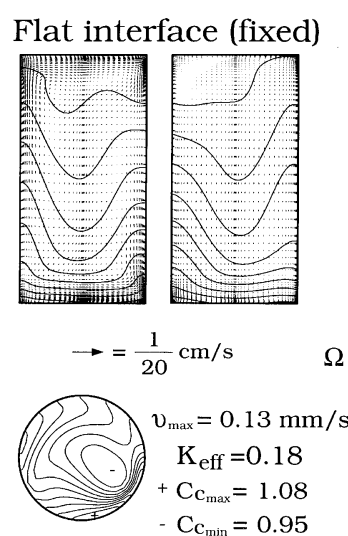

(a)
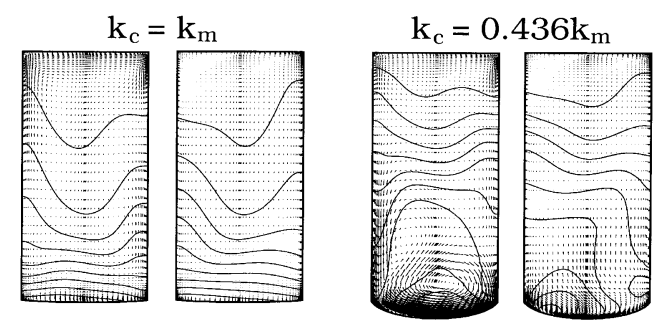

$\Omega=40 \mathrm{rpm} ; \mathrm{U}_{\mathrm{amp}}=-2 \times 10^{-4} \mathrm{~cm} / \mathrm{s}$

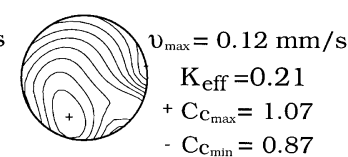

(b)

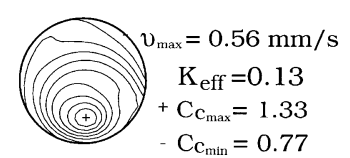

(c)

Fig. 6. Effects of interface shape on the flow and dopant fields $\left(40 \mathrm{rpm}, \theta=63.32^{\circ}\right)$ : (a) flat interface $\left(C_{\min }=2.48, C_{\max }=12.41\right)$; $(\mathrm{b})$ $k_{\mathrm{c}}=k_{\mathrm{m}} \quad\left(C_{\min }=1.93 ; C_{\max }=12.32\right) ; \quad(\mathrm{c}) \quad k_{\mathrm{c}}=0.436 k_{\mathrm{m}} \quad\left(C_{\min }=2.58, C_{\max }=15.27\right) ; \quad U_{\mathrm{amp}}=-2 \times 10^{-4} \mathrm{~cm} / \mathrm{s} \quad$ and $\quad \Delta C=$ $\left(C_{\max }-C_{\min }\right) / 10$. 


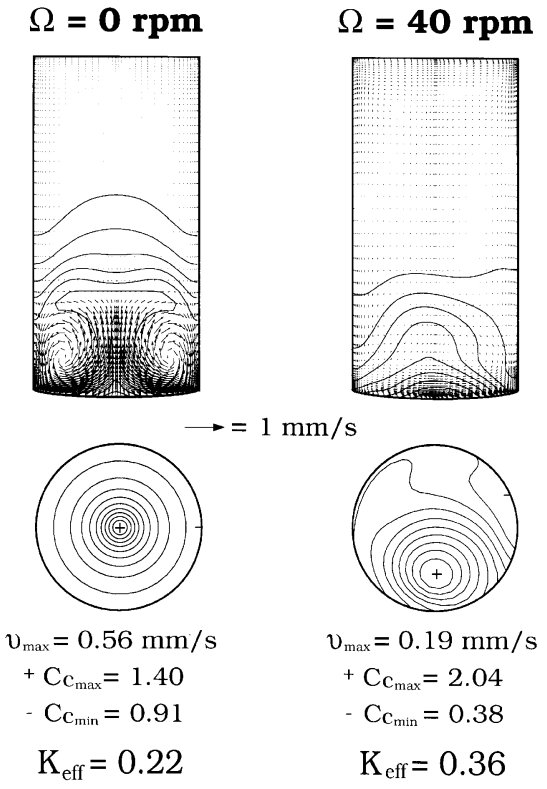

Fig. 7. Effects of rotation on flow and dopant fields for $k_{\mathrm{c}}=k_{\mathrm{m}}$ and $U_{\text {amp }}=-5.6 \times 10^{-4} \mathrm{~cm} / \mathrm{s}: \quad$ (a) $0 \mathrm{rpm} \quad\left(C_{\min }=1.02\right.$, $\left.C_{\max }=16.04\right) ; \quad\left(\right.$ b) $40 \mathrm{rpm} \quad\left(\theta=63.32^{\circ}, C_{\min }=1.01, C_{\max }=\right.$ 23.49).

the same, radial dopant segregation increases. More importantly, its dopant distribution is also quite different from that in Fig. 6a. As the interface concavity further increases with the use of a smaller $k_{\mathrm{c}}$, the convection near the growth interface increases, and the radial segregation increases further as well. But, the segregation pattern remains similar.

If we further take the case with $k_{\mathrm{c}}=k_{\mathrm{m}}$ for examination, but with a much higher growth rate $\left(5.6 \times 10^{-4} \mathrm{~cm} / \mathrm{s}\right)$, the results showing the effect of $40 \mathrm{rpm}$ rotation are illustrated in Fig. 7. Due to the larger growth rate, the calculated $K_{\text {eff's }}$ are also larger. As shown in Fig. 7, the global dopant mixing at $40 \mathrm{rpm}$ is reduced significantly as compared with that of no rotation. This result is similar to that observed in the experiment [1]. More interestingly, although our definition of $K_{\text {eff }}$ is different from the previous one [1], which used the best-fit value to the axial segregation profile, the ratio $\left(\left.K_{\text {eff }}\right|_{40 \mathrm{rpm}} /\left.K_{\text {eff }}\right|_{0 \mathrm{rpm}}\right)$ appears to be about the same (about 1.6). Besides the increase of $K_{\text {eff }}$, the rotation introduces a severe radial segregation,

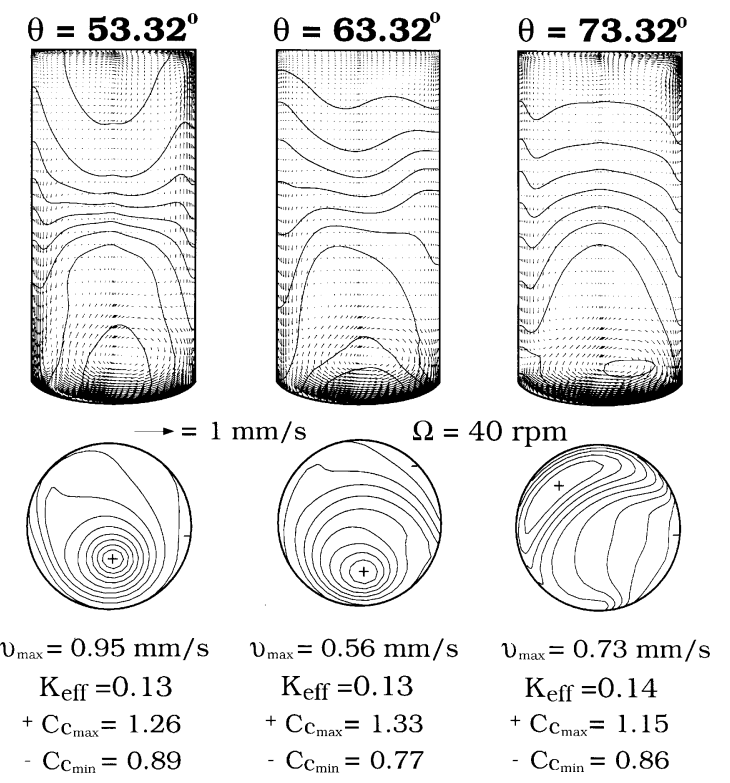

(b)

(c)

Fig. 8. Effects of tilt angle on flow and dopant fields: (a) $\theta=$ $53.32^{\circ}\left(C_{\min }=3.45, C_{\max }=14.42\right)$; (b) $\theta=63.32^{\circ}$ (free-swing angle) $\left(C_{\min }=2.58, C_{\max }=15.27\right)$; (c) $\theta=73.32^{\circ}\left(C_{\min }=2.91\right.$, $\left.C_{\max }=13.18\right) ; \Omega=40 \mathrm{rpm}$ and $U_{\mathrm{amp}}=-2 \times 10^{-4} \mathrm{~cm} / \mathrm{s}$.

i.e., more than $165 \%$. Therefore, the general feature of the free-swing rotation becomes clear. Before the centrifugal acceleration becomes dominant, the radial segregation increases with the rotation speed due to the poorer mixing. For the same interface concavity, increasing local convection seems to be the only way to reduce radial segregation, but they may reduce $K_{\text {eff }}$ due to the better global mixing. In addition to the further increase of rotation rate, one can also tilt the sample from the free-swing angle $\left(63.32^{\circ}\right)$.

For the free-swing configuration, the resultant gravity is antiparallel to the growth axis or the axial thermal gradient. Therefore, its flow intensity is supposed to be the weakest. Away from the freeswing angle, the convection increases. Fig. 8 shows the comparison of the flow and dopant fields at $40 \mathrm{rpm}$ for three different angles; the case in Fig. $5 b$ (at the same free-swing angle) is chosen for comparison (Fig. 8b). As shown in Figs. 8a and $\mathrm{c}$, the convection is enhanced with the tilting from 
the free-swing angle. As a result, due to the better global dopant mixing, $K_{\text {eff }}$ and radial segregation decrease. More importantly, with a smaller angle, the centrifugal acceleration is more perpendicular to the axial gradient. As a result, its effect is more obvious (similar to the horizontal one). On the other hand, besides the stronger convection, the larger tilt angle gives a different segregation pattern in the grown crystal.

\subsection{Rotation about the growth axis}

So far, we have illustrated the effects of rotation on the global (axial) and local (radial) dopant segregations. Indeed, operating the growth at the so-called magic-g level gives a weaker convection and thus poorer global mixing. As a result, one may expect a larger $K_{\text {eff }}$ and thus smaller axial segregation (if the growth distance is long enough, i.e. much greater than $D / U_{\mathrm{amp}}$ ). Unfortunately, we have found that the radial segregation at this critical rotation rate turns out to be larger due to the poorer local dopant mixing near the growth interface. Therefore, if one wants to control the growth with a small radial dopant segregation, while keeping a larger $K_{\text {eff }}$, he needs to control the growth interface to be flat or convex. Indeed, this is feasible by modifying the heating profile. Recently, Lan [7] has shown numerically using a $2 \mathrm{D}$ model that rotating the sample about the growth axis may be a better way to keep a high $K_{\text {eff }}$ value while reducing radial segregation. Since in his study the axial gradient is large, being $50^{\circ} \mathrm{C} / \mathrm{cm}$, the centrifugal acceleration can contribute more. In our case here, the axial thermal gradient is smaller $\left(10^{\circ} \mathrm{C} / \mathrm{cm}\right)$, but the diameter is twice as large as his. Therefore, we anticipate that a similar result may be obtained.

Fig. 9 shows the effects of rotation rate for this configuration. As shown, flow is suppressed most effectively at about $80 \mathrm{rpm}$. In fact, even at only $40 \mathrm{rpm}$, this configuration is more efficient than the free-swing one in flow suppression; the highest melt velocity is $0.12 \mathrm{~mm} / \mathrm{s}$ in Fig. 9a, as compared with $0.56 \mathrm{~mm} / \mathrm{s}$ in Fig. 5 b. More interestingly, at $80 \mathrm{rpm}$ shown in Fig. 9b, the dopant distribution is almost the same as the diffusion-controlled one. On increasing the rotation speed to $160 \mathrm{rpm}$, the

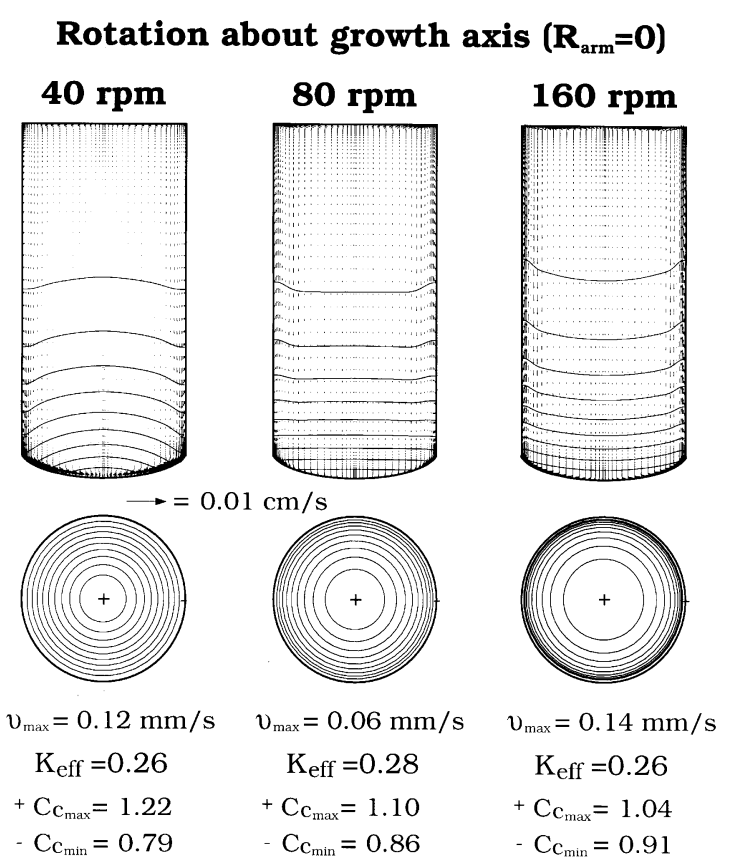

Fig. 9. Effects of rotation rate on flow and dopant fields for rotation about growth axis: (a) $40 \mathrm{rpm} \quad\left(C_{\min }=1.24\right.$, $\left.C_{\max }=14.05\right) ; \quad(\mathrm{b}) \quad 80 \mathrm{rpm} \quad\left(C_{\min }=1.22, C_{\max }=12.59\right)$; (c) $160 \mathrm{rpm} \quad\left(C_{\min }=1.28, C_{\max }=11.91\right) ; \quad U_{\mathrm{amp}}=-2 \times$ $10^{-4} \mathrm{~cm} / \mathrm{s}$.

flow speed increases indicating that the centrifugal acceleration becomes important near the ampoule wall. The radial segregation is improved, while $K_{\text {eff }}$ only decreases slightly.

In Fig. 10, we put the maximum melt speeds at different rotation rates from the previous calculations together. We can find that rotating the sample about the growth axis indeed suppresses the convection more effectively than the free-swing one. The slope of the melt speed versus rotation rate is -1 (or $-\frac{1}{2}$ in terms of Ta number). This scaling is also shown numerically by Lan [7] for the damping effect by the Coriolis force. The weakest flow for axial rotation appears at about $80 \mathrm{rpm}$. The corresponding radial segregation (left scale) and $K_{\text {eff }}$ (right scale) are shown in Fig. 11. Again, rotation about the growth axis gives a smaller radial segregation, but larger $K_{\text {eff }}$. The slow reduction of $K_{\text {eff }}$ for the rotation about the growth axis is also due to the much smaller acceleration contribution in this configuration. Therefore, it 


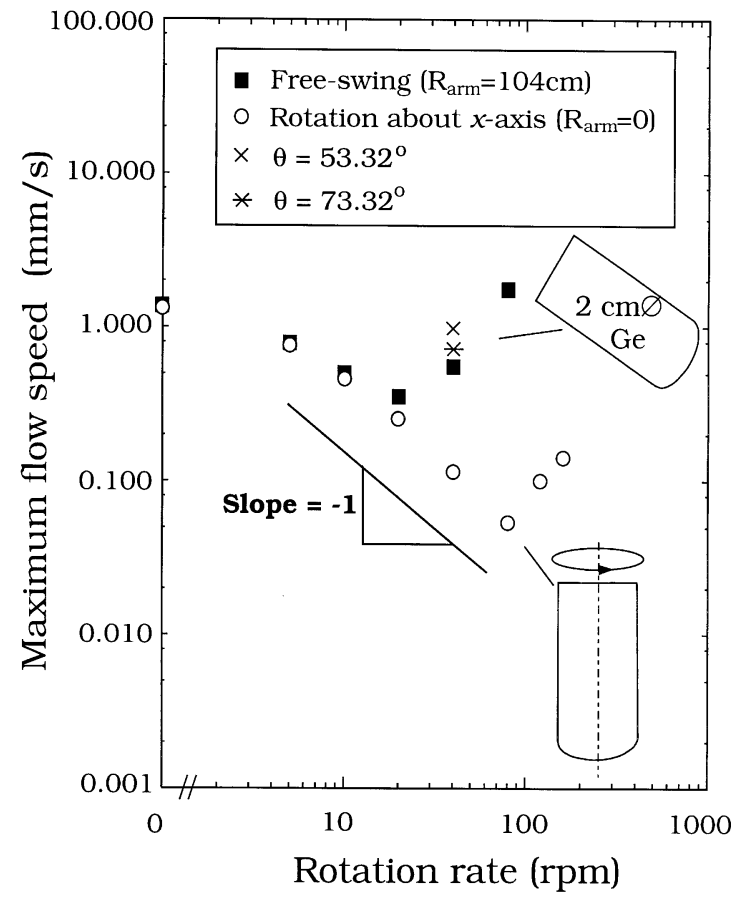

Fig. 10. Effects of rotation rate on the maximum melt velocity for the free-swing and rotation-about-axis $\left(R_{\text {arm }}=0 \mathrm{~cm}\right.$ and $\theta=0^{\circ}$ ) configurations; the ones from Fig. 2 have a smaller interface deflection.

may be concluded that rotating the system about the growth axis may be a better way for the operation if one wants to take the advantages of the centrifuge.

\section{Conclusions}

In this study, we performed for the first time a self-consistent simulation of gradient-freeze crystal growth in a centrifuge. In addition to the heat transfer and melt flow, the interface shape and radial dopant segregation are also considered for various growth configurations. Detailed benchmarkings with previous calculations are performed and good agreement is found. For the free-swing configuration, increasing rotation rate suppresses convection before the centrifugal acceleration kicks in. For the horizontal configuration, the

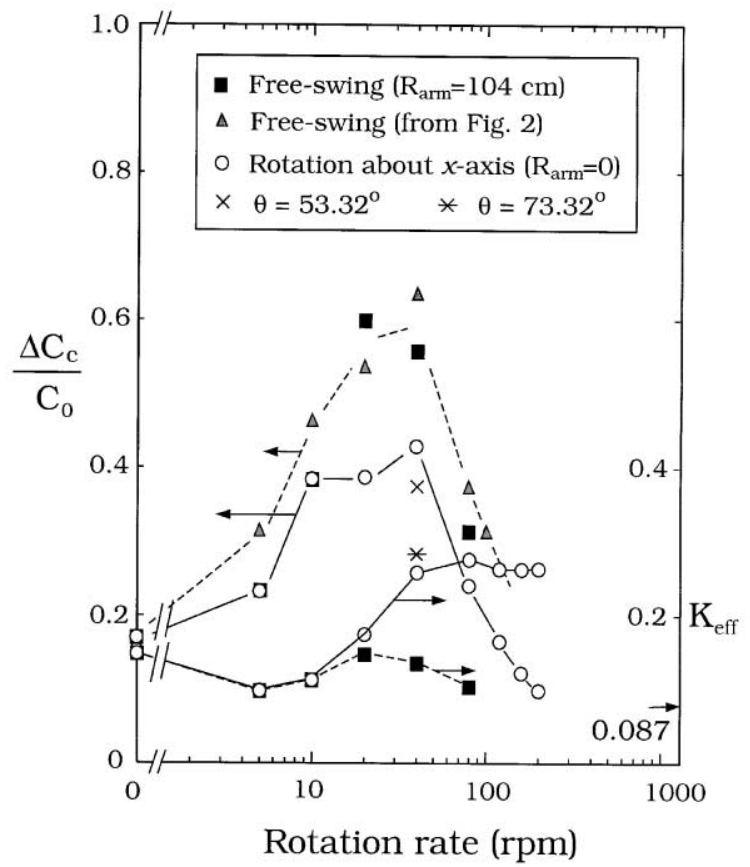

Fig. 11. Effects of rotation rate on radial segregation (left scale) and effective segregation coefficients (right scale) for the free-swing and rotation-about-axis configurations; $\Delta C_{\mathrm{c}}=$ $\left(C_{\mathrm{c}_{\max }}-C_{\mathrm{c}_{\min }}\right)$. The ones from Fig. 2 have a smaller interface deflection.

flow speed decreases monotonically with the rotation speed and as a result, the unstable flow is suppressed. Especially, for the free-swing configuration, a condition for a minimum convection is found, where the Coriolis force balances the gravitational ones. Although a larger effective segregation coefficient is found at the critical rotation speed, the poorer mixing near the growth interface can induce severe radial segregation. Minimizing the radial segregation would require reducing the interface concavity or the growth rate. Interestingly, we have found that rotating the system about the growth axis may be a better way to control both the axial and radial segregation for the growth with a concave interface and this is also consistent with previous 2D calculations. Nevertheless, further numerical simulation for the whole growth as well as the experimental verification is necessary, which will be carried out in the near future. 


\section{Acknowledgements}

The authors are grateful for the support from the National Science Council and the National Center for High Performance Computing of the Republic of China under Grant No. 89-2214-E002-040. CWL also acknowledges the fruitful discussion with Profs. W.R. Wilcox and L.L. Regel at the beginning of this study.

\section{References}

[1] J. Friedrich, J. Baumgartl, H.-J. Leister, G. Müller, J. Crystal Growth 167 (1996) 45.

[2] H. Rodot, L.L. Regel, G.V. Sarafanov, H. Hamidi, I.V. Videskii, A.M. Turtchaninov, J. Crystal Growth 79 (1986) 77.

[3] G. Müller, G. Neumann, W. Weber, J. Crystal Growth 129 (1992) 8.

[4] H. Rodot, L.L. Regel, A.M. Turtchaninov, J. Crystal Growth 104 (1990) 280.
[5] W.A. Arnold, W.R. Wilcox, F. Carlson, A. Chait, L.L. Regel, J. Crystal Growth 129 (1992) 24.

[6] W.R. Wilcox, L.L. Regel, W.A. Arnold, J. Crystal Growth 187 (1998) 543.

[7] C.W. Lan, Effects of centrifugal acceleration on the flows and segregation in vertical Bridgman crystal growth by steady ampoule rotation, J. Crystal Growth 229 (2001) 595.

[8] C.W. Lan, M.C. Liang, J.H. Chian, J. Crystal Growth 212 (2000) 340.

[9] P.M. Adornato, R.A. Brown, J. Crystal Growth 80 (1987) 155.

[10] C.W. Lan, F.C. Chen, Comput. Methods Appl. Mech. Eng. 131 (1996) 191.

[11] C.W. Lan, J. Crystal Growth 197 (1999) 983.

[12] M.C. Liang, C.W. Lan, J. Comput. Phys. 127 (1996) 330.

[13] C.W. Lan, M.C. Liang, J. Comput. Phys. 152 (1999) 55.

[14] M.C. Liang, C.W. Lan, J. Crystal Growth 167 (1996) 320.

[15] D. Jansinski, A.F. Witt, J. Crystal Growth 71 (1985) 295.

[16] J. Friedrich, Ph.D. Dissertation, University Erlangen, Nürnberg, Germany, 1996.

[17] G. Müller, A. Ostrogorsky, in: D.T.J. Hurle (Ed.), Handbook of Crystal Growth 2b: Growth Mechanisms and Dynamics, North-Holland, Amsterdam, 1994, p. 754. 